\title{
INTRODUCTION \\ Deep brain stimulation: current assessment, new applications, and future innovations
}

\author{
Kim Burchiel, MD, ${ }^{1}$ Mark A. Liker, MD, ${ }^{2}$ and Andres M. Lozano, MD, PhD ${ }^{3}$ \\ ${ }^{1}$ Department of Neurological Surgery, Oregon Health \& Science University, Portland, Oregon; ${ }^{2}$ Department of Neurosurgery, \\ Keck Hospital, University of Southern California, Los Angeles, California; and ${ }^{3}$ Division of Neurosurgery, University of Toronto, \\ Toronto Western Hospital, Toronto, Ontario, Canada
}

Deep brain stimulation (DBS) for the treatment of movement disorders has become part of the fabric of neurological surgery over the past 25 years. Other indications for DBS, such as for the treatment of depression and epilepsy, are currently being explored and developed. And many other potential uses of DBS have been suggested. The goals of this issue of Neurosurgical Focus are to illuminate new knowledge of DBS with regard to clinical outcomes, to describe new applications for this technology, and to suggest future directions for this extraordinary surgical therapy. A combination of factors has driven the exploration into new avenues of intervention for DBS, including improved safety, more precise targeting techniques, a better comprehension of neural pathways underlying certain pathologies, and, perhaps most importantly, heightened societal acceptance of DBS surgery. Moving beyond the mainstay of Parkinson's disease (PD), essential tremor, and dystonia, we are now exploring more effective interventions into diseases that affect even larger portions of the population. We hope that the brief summaries below will assist the reader in finding articles of particular interest.

This issue provides a comprehensive review of the history, current state, and potential future applications of DBS for psychiatric disorders by highlighting psychiatric conditions and their associated target substrates. This includes the full complement of associated investigations into specific psychiatric diseases. Deep brain stimulation may also be helpful in rare conditions like Kleefstra syndrome, a malady that has aspects of obsessive-compulsive disorder and Tourette's syndrome.

An experience that has puzzled many involved in DBS treatment is post-DBS psychosis, which can develop months or years after surgery. In this issue of Focus, an important study from Johns Hopkins suggests that while postoperative psychosis in PD patients implanted with DBS systems is "widely incident" (28\%), its occurrence is likely independent of the implantation of DBS electrodes or the DBS therapeutic settings.

In their paper, Sinha et al. discuss the possibility of DBS for severe autism. This increasingly recognized and disabling disorder does not have much traction in the neurosurgical field yet. Advances in the understanding of autism and its pathophysiology suggest that there may be some opportunities to regulate dysfunctional circuits within this heterogeneous disorder.

The potential role of DBS as it relates to epilepsy is detailed in 2 articles. The paper by Gummadavelli and colleagues tackles the important issue of loss of consciousness that can occur in either primary generalized epilepsies or partial epilepsies with secondary generalization. The loss of consciousness that occurs with epilepsy is most disabling. Measures to understand the pathophysiology of the loss of consciousness and to develop preventative or palliative measures are highly sought after and described in this work. Van Gompel and colleagues have studied recordings in the hippocampus in the context of anterior nuclear subthalamic stimulation in 2 patients. These observations suggest the possibility of constructing a closedloop epilepsy stimulation system for seizure control.

Deep brain stimulation for chronic pain enjoyed a heyday in the 1980s, and its application in this area has waned dramatically since then. However, there remains a potential for DBS of the dorsal anterior cingulate cortex in the treatment of chronic neuropathic pain, believed to affect $3 \%-4.5 \%$ of the worldwide population. Given its critical 
role in cognitive and affective processing, this target may provide improved therapeutic benefit in this complex but disruptive disease entity.

Obesity is one of the major factors contributing to increasing health care costs in the US. Ho et al. describe the rationale for neuromodulation as an alternative to bariatric surgery in certain patients and define the structure of a DBS trial for obesity. Complementing this paper, Dupré et al. emphasize potential targets and past, present, and future possibilities in treating obesity with DBS to modulate caloric intake and energy expenditure.

An estimated $38 \%$ of patients with essential tremor also have essential vocal tremor (VT), and many other neuro- logical disorders are associated with VT. Ho et al. describe a novel multidisciplinary technique to target improvement in VT using intraoperative otolaryngological adjuncts.

From the National (Nationwide) Inpatient Sample database we learn that the hospital caseload volume of DBS implantation surgeries influences quality. Specifically, centers with a higher volume of DBS procedures ( $>5 /$ year) have better postoperative outcomes.

This issue of Neurosurgical Focus should be of interest both to those of us currently engaged with DBS implantation therapy and to those who wish to explore one of the most exciting forefronts of our discipline.

http://thejns.org/doi/abs/10.3171/2015.4.FOCUS15179 